\title{
An Algebraic Weak Factorisation System on 01-Substitution Sets: A Constructive Proof
}

\begin{abstract}
ANDREW SWAN
Abstract: We will construct an algebraic weak factorisation system on the category of 01 -substitution sets such that the R-algebras are precisely the Kan fibrations together with a choice of Kan filling operation. The proof is based on Garner's small object argument for algebraic weak factorisation systems. In order to ensure the proof is valid constructively, rather than applying the general small object argument, we give a direct proof based on the same ideas. We use the resulting awfs and the notion of path object to explain why the $J$-computation rule is absent from the original cubical set model. We will define an alternative path object, which can be used to implement the $J$-computation rule in cubical sets.
\end{abstract}

2010 Mathematics Subject Classification 03F50, 03F55, 55U35 (primary); 03F60, 03B15 (secondary)

Keywords: cubical sets, nominal sets, algebraic weak factorisation systems, constructive mathematics, type theory

\section{Introduction}

\subsection{Aims}

Pitts showed in [12] and [13], following earlier work by Staton, that the category of cubical sets is equivalent to a category based on nominal sets, called the category of 01 -substitution sets, or 01Sub.

We will construct an algebraic weak factorisation system on 01Sub such that the Ralgebras are precisely the Kan fibrations together with a choice of Kan filling operation. It will be algebraically free in the sense used by Garner in [7]. However rather than applying the result in [7], we will give a direct construction based on the same ideas. The construction is also similar to Kan completion, as referred to in Bezem, Coquand and Huber [3]. This approach has three main advantages.

Firstly, this allows us to ensure that the proof holds in a constructive setting (such as the set theory $\mathbf{C Z F}$ ), whereas it is not clear whether the proof in [7] is valid constructively. 
Secondly, it allows us to see explicitly the objects involved in the construction, which may be useful if one wanted to use the ideas here in computer implementations.

Thirdly, we will see that the proof uses only countable colimits, not requiring the full cocompleteness of 01Sub. Although 01Sub is cocomplete, this property should make it easier to apply the proof in other contexts. For example, if one were to define "01-substitution assemblies" by analogy with Stekelenburg's recent work on simplicial assemblies in [16] this may be useful.

We will use the awfs to give an explanation why the $J$-computation rule is absent in the original Bezem-Coquand-Huber cubical set model in [3], by showing that a certain map cannot be proved constructively to be a left map in the awfs. We will show however, that with a more sophisticated definition one can construct stable path objects in 01 -substitution sets. In a future paper, the author will show how these new path objects can be understood via Riehl's notion of algebraic model structure (from [15]) and give a more complete proof that this gives a model of type theory including the computational rule for identity. These ideas (via an earlier version of this paper and direct communication with the author), together with some rephrasing and simplification have already been used by Cohen, Coquand, Huber and Mörtberg to implement identity types in a new variant of cubical sets (see [6, Section 9.1]).

\subsection{Cubical Sets and 01-Substitution Sets}

Cubical sets were developed by Bezem, Coquand and Huber in [3] (and described in more detail in Huber [9]) as a constructive model of homotopy type theory, inspired by the simplicial set model due to Voevodsky and other homotopical models.

In this paper we will work over this category, 01Sub of 01 -substitution sets, using the notation and definitions of open box and Kan fibration that appear in Pitts [12]. For a good introduction to nominal sets, on which 01-substitution sets are based, see Pitts [11].

We recall the following definitions from [11]. Let $\mathbb{A}$ be a set (which we will refer to as the set of names). Write $\operatorname{Perm}(\mathbb{A})$ for the group of finite permutations (that is, permutations $\pi$ such that $\pi(a)=a$ for all but finitely many $a \in \mathbb{A})$. Recall that a $\operatorname{Perm}(\mathbb{A}$ )-set is a set $X$, together with an action of $\operatorname{Perm}(\mathbb{A})$ on $X$ (or equivalently a presheaf over Perm $(\mathbb{A})$ when viewed as a one object category in the usual way). 
Definition 1.1 (Pitts, Gabbay)

(1) Let $X$ be a Perm(A)-set (writing for the action) and let $x \in X$. We say $A \subseteq \mathbb{A}$ is a support for $x$ if whenever $\pi(a)=a$ for all $a \in A$, also $\pi \cdot x=x$.

(2) Let $X$ be a Perm(A)-set and $x \in X$. We say $x$ is equivariant if $\pi \cdot x=x$ for all $\pi \in \operatorname{Perm}(\mathbb{A})$, (or equivalently if $\emptyset$ is a support for $x$ ).

(3) Let $X$ and $Y$ be Perm(A)-sets. A function $f: X \rightarrow Y$ is equivariant if it is a morphism in the category of $\operatorname{Perm}(\mathbb{A})$ sets, or equivalently if it is equivariant as an element of the exponential $Y^{X}$ in the category of $\operatorname{Perm}(\mathbb{A})$ sets, which is described explicitly as the set of functions $X$ to $Y$ with action given by conjugation.

(4) A nominal set is a Perm $(\mathbb{A})$-set $X$, such that for every $x \in X$, there exists a finite set $A \subseteq \mathbb{A}$ such that $A$ is a support for $x$.

(5) Let $X$ and $Y$ be nominal sets and let $x \in X$ and $y \in Y$. We say $x$ is fresh for $y$ and write $x \# y$ if there exist finite sets $A, B \subseteq \mathbb{A}$ such that $A$ is a support for $x, B$ is a support for $y$ and $A \cap B=\emptyset$.

(6) Let $X$ and $Y$ be nominal sets. The separated product, $X * Y$, of $X$ and $Y$ is the nominal set with elements $(x, y)$ where $x \in X, y \in Y$ and $x \# y$ (with the action defined componentwise).

(7) Let $X$ be a nominal set. We define an equivalence relation $\sim$ on $\mathbb{A} \times X$, referred to as $\alpha$-equivalence as follows. $(a, x) \sim\left(a^{\prime}, x^{\prime}\right)$ if and only there exists $a^{\prime \prime}$ fresh for $a, x, a^{\prime}, x^{\prime}$ such that $\left(a^{\prime \prime} a\right) \cdot x=\left(a^{\prime \prime} a^{\prime}\right) \cdot x^{\prime}$ (where $(a b)$ is the finite permutation swapping $a$ and $b$ and fixing all other names). The quotient $\mathbb{A} \times X / \sim$ can be viewed in a natural way as a nominal set $[\mathbb{A}] X$, referred to as the name abstraction on $X$. We write $\langle a\rangle x$ for the equivalence class of $(a, x)$.

The lemma below is a variant of standard results in nominal sets as in [11, Chapter 4]. It allows us to easily construct morphisms $F: W \rightarrow Y$ when $W \subseteq[\mathbb{A}] X$ for some nominal sets $X$ and $Y$ by the following heuristic. We assume we have been given some finite list of names. We then define $F(\langle a\rangle x)$ when $a$ is fresh for the given names, to get a partial function on the preimage of $W$ in $\mathbb{A} \times X$. This can be done without worrying whether or not $F$ respects $\alpha$-equivalence (a priori). We then check that $a$ is fresh for $F(\langle a\rangle x)$. We then check or note by inspection that $F$ is equivariant modulo the names we were given (ie the given list of names is a support for $F$ ). We then apply the lemma to get a well defined morphism, that in particular necessarily respects $\alpha$-equivalence by well definedness.

Lemma 1.2 Let $Z$ be a subobject of $\mathbb{A} \times X$ (ie an equivariant subset) and write $Z^{\prime}$ for the image of $Z$ under the projection $\mathbb{A} \times X \rightarrow[\mathbb{A}] X$. Let $Y$ be another nominal set and 
$F: Z \rightarrow Y$ a partial function with finite support (under the action given by conjugation) such that for any $a \# F$, if $(a, x) \in Z$, then $F(a, x) \downarrow$ and $a \# F(a, x)$. Then $F$ extends uniquely to a function $\bar{F}: Z^{\prime} \rightarrow Y$. Furthermore, $\bar{F}$ is constructed equivariantly, in the following sense: for any $\pi \in \operatorname{Perm}(\mathbb{A}), \overline{\pi \cdot F}=\pi \cdot \bar{F}$.

Proof This is a slight generalisation of Pitts [11, Theorem 4.15] and the same proof works here.

We will also use the following corollary.

Corollary 1.3 Let $Z$ and $Z^{\prime}$ be the same as in Lemma 1.2. Let $W$ be another nominal set, and let $F: W \times Z \rightarrow Y$ be a partial function with finite support $A \subseteq \mathbb{A}$ such that for any $(a, x) \in Z$ and $w \in W$, if $a \# F$ and $a \# w$ then $F(a, x) \downarrow$ and $a \# F(w,(a, x))$. Then $F$ extends uniquely to a map $\bar{F}: W \times Z^{\prime} \rightarrow Y$ and $A$ is a support for $\bar{F}$.

Proof For each $w \in W$, we have a partial function $F(w,-): Z \rightarrow Y$. Furthermore, if $B$ is a finite support for $w$, then $A \cup B$ is a finite support for $F(w,-)$. Applying Lemma 1.2 gives us a function $\bar{F}_{w}: Z^{\prime} \rightarrow Y$. We then define $F: W \times Z^{\prime} \rightarrow Y$ by $\bar{F}(w, z):=\bar{F}_{w}(z)$.

We now recall from [12] and [13] Pitts' definition of 01 -substitution sets and his translation of the Bezem-Coquand-Huber definitions of open box and Kan fibration.

Definition 1.4 (Pitts) Let $X$ be a nominal set. A 01 -substitution operation on $X$ is a morphism $s: X \times \mathbb{A} \times 2 \rightarrow X$ in nominal sets, satisfying the following axioms. We write $x(a:=i)$ for $s(x, a, i)$. For any $x \in X, a, a^{\prime} \in \mathbb{A}$ and $i, i^{\prime} \in 2$,

(1) $a \# x(a:=i)$

(2) if $a \# x$, then $x(a:=i)=x$

(3) if $a \neq a^{\prime}$, then $x(a:=i)\left(a^{\prime}:=i^{\prime}\right)=x\left(a^{\prime}:=i^{\prime}\right)(a:=i)$

We say that a nominal set equipped with a 01 -substitution operation is a 01 -substitution set. 01 -substitution sets form a category 01Sub, where morphisms are morphisms in nominal sets that also preserve the 01 -substitution operation.

Note that the name abstraction $[\mathbb{A}] X$ of a 01 -substitution set $X$ can be viewed itself as a 01 -substitution set in a canonical way.

We now give the definitions of open box and Kan fibration. 
Definition 1.5 (Pitts)

(1) Let $A$ be a finite subset of $\mathbb{A}$ with $a \in A$ and let $f: X \rightarrow Y$ be a morphism in 01Sub. A 1-open $(A, a)$-box relative to $f$ is a pair $(u, y)$ with $y \in Y$ and $u:(A \times 2) \backslash\{(a, 1)\} \rightarrow X$ satisfying the following. For all $(b, i),\left(b^{\prime}, i^{\prime}\right) \in$ $(A \times 2) \backslash\{(a, 1)\}$,
(a) $b \# u(b, i)$
(b) $u(b, i)\left(b^{\prime}:=i^{\prime}\right)=u\left(b^{\prime}, i^{\prime}\right)(b:=i)$
(c) $f(u(b, i))=y(b:=i)$

(2) We also define 0 -open $(A, a)$-box relative to $f$ by simply replacing 1 with 0 in the above definition.

(3) Let $(u, y)$ be a 1-open $(A, a)$-box relative to $f$. A filler for $(u, y)$ is $x \in X$ such that

(a) for all $(b, i) \in(A \times 2) \backslash\{(a, 1)\}, x(b:=i)=u(b, i)$

(b) $f(x)=y$

(4) We similarly define fillers for 0-open boxes.

(5) Let $f: X \rightarrow Y$. A Kan filling operator for 1-open boxes is for each 1-open box $(u, y)$, a choice of filler, $f \uparrow(u, y)$ satisfying the following conditions (which we refer to as uniformity conditions).

(a) For each finite permutation $\pi, \uparrow(\pi(u, y))=\pi(\uparrow(u, y))$.

(b) Whenever $(u, y)$ is a 1 -open $(A, a)$-box with $c \# A$ and $i \in 2$, we have $\uparrow((u, y)(c:=i))=(\uparrow(u, y))(c:=i)$.

We will sometimes write $f \uparrow(u, y)$ just as $\uparrow(u, y)$. If $u$ is a box in direction $a$, we will write $f^{+}(u, y)$ to mean $(f \uparrow(u, y))(a:=1)$.

(6) We similarly define the notion of Kan filling operator $\downarrow(u, y)$ for 0 -open boxes $(u, y)$.

(7) We say $f$ is a Kan fibration (or simply fibration) if it admits both Kan filling operators.

Remark 1.6 Note that the definition of open box includes the case where $A=\{a\}$, and so the "box" only consists of one point $x \in X$ and an element of $Y$. This is an important special case, and essentially says that any path in $Y$ with endpoint $f(x)$ can be lifted to a path in $X$ with endpoint $x$. 
Remark 1.7 Note that as a special case of the uniformity conditions we have that whenever $\pi$ is a permutation of a finite set $A \subseteq \mathbb{A}$ that fixes $a \in A$ and $u$ is a 1 -open (A,a)-box we have that if $\pi \cdot u=u$ then $\pi(\uparrow u)=\uparrow u$. Hence the uniformity conditions provide conditions on each filler individually, not just conditions on how the fillers of different open boxes relate to each other. In classical logic with the axiom of choice, the existence of uniform Kan filling operators is equivalent to the existence of fillers satisfying this "symmetry preserving" condition, which is stronger than just requiring fillers without the condition.

\subsubsection{A Note on Nominal Sets in a Constructive Setting}

As in Bezem, Coquand and Huber [3] we require the assumption that the set $\mathbb{A}$ of names has decidable equality. Note in particular that for sets with decidable equality, finite and finitely enumerable subsets coincide (ie, if a subset of $\mathbb{A}$ is the image of a function from a natural number to $\mathbb{A}$, then it is the image of an injection from a natural number to $\mathbb{A}$ ) and these are decidable subsets (ie, if $A \subseteq \mathbb{A}$ is finite then every element of $\mathbb{A}$ belongs to $A$ or does not). We also assume that $\mathbb{A}$ is infinite in the strong sense that for every finite $A \subseteq \mathbb{A}$ there exists $a \in \mathbb{A}$ such that $a \notin A$.

In Pitts [11] heavy use is made of the existence of least finite support. Constructively this can't be guaranteed to exist; the proof of Theorem 6.5 provides an example where the freshness relation is not decidable and hence we cannot show that every element has least finite support. In fact the intersection of all finite supports can fail to be finite and can fail to be a support (see Swan [17]). In practice, however, most of the uses of least finite support in [11] can be viewed as a "notational device" to make definitions and statements of theorems more concise, and these can be rephrased to work in a constructive setting (see the work by Choudhury in [5]).

In some places we will use the notion of nerve of a metric space. For these we will assume for convenience the axiom of dependent choice. However, aside from those places and in particular for the main results, no choice will be required.

\subsubsection{The Nerve of a Complete Metric Space}

To help us give some non trivial examples of objects of 01Sub later, we will use the notion from homotopical algebra of nerve. This gives a way to construct 01 -substitution sets from topological spaces. 
For this subsection suppose that $\mathbb{A}=\mathbb{N}$. Also, for convenience we will assume the axiom of dependent choice, since it is often implicitly assumed in Bishop style analysis that we use here.

Note that $[0,1]^{\mathbb{N}}$ has a canonical metric given by the product metric (see Bishop and Bridges [4, Chapter 4, definition 1.7]), defined as follows for $r, r^{\prime} \in[0,1]^{\mathbb{N}}$.

$$
d\left(r, r^{\prime}\right):=\sum_{n \in \mathbb{N}} 2^{-n}\left|r(n)-r^{\prime}(n)\right|
$$

Let $X$ be a metric space. Then we define an action on uniformly continuous functions $F:[0,1]^{\mathbb{N}} \rightarrow X$ as follows. For $\pi \in \operatorname{Perm}(\mathbb{A})$ and $r \in[0,1]^{\mathbb{N}}$ :

$$
(\pi \cdot F)(r):=F(r \circ \pi)
$$

We then define the nerve of $X, N(X)$ to be the subobject of the Perm $(\mathbb{A})$ set defined above consisting of elements that have finite support. Note that this is a nominal set by definition.

Lemma 1.8 Let $A$ be a finite subset of $\mathbb{N}$. Then for $F \in N(X), A$ is a support for $F$ if and only if for every $r, r^{\prime} \in[0,1]^{\mathbb{N}}$ if $\left.r\right|_{A}=\left.r^{\prime}\right|_{A}$ then $F(r)=F\left(r^{\prime}\right)$.

Proof Assume first that $A$ is a support for $F$. We need to show that for $r, r^{\prime} \in[0,1]^{\mathbb{N}}$ with $\left.r\right|_{A}=\left.r^{\prime}\right|_{A}$, we have $F(r)=F\left(r^{\prime}\right)$. Note that it suffices to show that for all $\epsilon>0$, $d\left(F(r), F\left(r^{\prime}\right)\right)<\epsilon$ (for instance by [4, Chapter 2, Lemma 2.18]).

For any $\epsilon>0$, there exists by uniform continuity of $F, \delta>0$ such that for all $x, y \in[0,1]^{\mathbb{N}}$ if $d(x, y)<\delta$ then $d(F(x), F(y))<\frac{\epsilon}{2}$. Let $N$ be such that $2^{-N}<\delta$ and such that $n<N$ for all $n \in A$. Define $B$ to be $\{0, \ldots, N\} \backslash A$ and let $\left\{b_{1}, \ldots, b_{k}\right\}$ be an enumeration of $B$, with $b_{i} \neq b_{j}$ for $i \neq j$.

Then define $\pi$ to be the composition of transpositions

$$
\pi:=\left(\begin{array}{lll}
b_{1} & N+1)\left(b_{2}\right. & N+2
\end{array}\right) \ldots\left(\begin{array}{ll}
b_{k} & N+k
\end{array}\right)
$$

and define $r^{\prime \prime} \in[0,1]^{\mathbb{N}}$ by

$$
r^{\prime \prime}(n):= \begin{cases}r(n) & n \leq N \text { or } n>N+k \\ r^{\prime}\left(b_{i}\right) & n=N+i \text { for some } 1 \leq i \leq k\end{cases}
$$

Then note that $r$ and $r^{\prime \prime}$ agree on the set $\{0, \ldots, N\}$, so by the definition of the metric on $[0,1]^{\mathbb{N}}$ we have that $d\left(r, r^{\prime \prime}\right) \leq 2^{-N}<\delta$. Similarly, we have $d\left(r^{\prime}, r^{\prime \prime} \circ \pi\right) \leq 2^{-N}<\delta$. Further, since $\pi$ fixes $A$, which is a support for $F$, we have

$$
\begin{aligned}
F\left(r^{\prime \prime} \circ \pi\right) & =(\pi \cdot F)\left(r^{\prime \prime}\right) \\
& =F\left(r^{\prime \prime}\right)
\end{aligned}
$$


Therefore we have

$$
\begin{aligned}
d\left(F(r), F\left(r^{\prime}\right)\right) & \leq d\left(F(r), F\left(r^{\prime \prime}\right)\right)+d\left(F\left(r^{\prime \prime} \circ \pi\right), F\left(r^{\prime}\right)\right) \\
& <\frac{\epsilon}{2}+\frac{\epsilon}{2} \\
& =\epsilon
\end{aligned}
$$

as required.

Finally, the converse is easy to show by noting that if $\pi$ fixes $A$ then $\left.r\right|_{A}=\left.(r \circ \pi)\right|_{A}$ for all $r \in[0,1]^{\mathbb{N}}$.

We now define a substitution operation on $N(X)$ as follows. For each $F \in N(X), a \in \mathbb{N}$ and $i \in\{0,1\}$, we first define $r(a:=i)(b)$ for $r \in[0,1]^{\mathbb{N}}$ and $b \in \mathbb{N}$ by

$$
r(a:=i)(b):= \begin{cases}r(b) & b \neq a \\ i & b=a\end{cases}
$$

We then define $F(a:=i)$ by

$$
F(a:=i)(r):=F(r(a:=i))
$$

One can use Lemma 1.8 to show that this satisfies the axioms for 01 -substitution set.

Also note that we can use Lemma 1.8 to prove the following lemma, that we will use later.

\section{Lemma 1.9}

(1) For any $a \in \mathbb{N}$ there is a correspondence between $F \in N(X)$ such that $\{a\}$ is a support for $F$ and uniformly continuous functions $[0,1] \rightarrow X$.

(2) Such an $F$ corresponds to a constant function $[0,1] \rightarrow X$ if and only if $\emptyset$ is a support for $F$.

Proposition 1.10 Let $X$ be a complete metric space. Then the unique map $N(X) \rightarrow 1$ is a fibration. (We say that $N(X)$ is fibrant.)

Proof We are given an open box $u: A \times 2 \backslash(a, 1)$ for some finite set $A \subseteq \mathbb{N}$ and $a \in A$ and we need to construct a filler in such a way that this can be done uniformly.

For each $(b, i) \in A \times 2$, define $X_{b, i} \subseteq[0,1]^{A}$ by

$$
U_{b, i}:=\left\{r \in[0,1]^{A} \mid r(b)=i\right\}
$$


Note that we can easily view $u$ as a uniformly continuous function

$$
\bar{u}: \bigcup_{(b, i) \in A \times 2 \backslash(a, 1)} U_{b, i} \times[0,1]^{\mathbb{N} \backslash A} \rightarrow X
$$

Note that one can piecewise linearly define a uniformly continuous retraction, $\tau$ from a dense subset $D$ of $[0,1]^{A}$ to $\bigcup_{(b, i) \in A \times 2 \backslash(a, 1)} U_{b, i}$. We then define a function

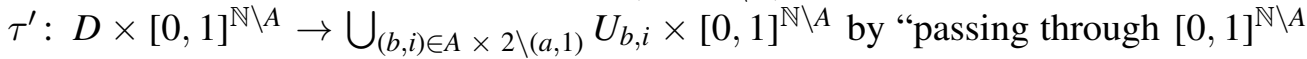
unchanged." Then the composition $\bar{u} \circ \tau^{\prime}$ is a uniform continuous function from a dense subset of $[0,1]^{\mathbb{N}}$ to a complete metric space. Under these conditions one can extend $\bar{u} \circ \tau$ to a uniformly continuous function $[0,1]^{\mathbb{N}} \rightarrow X$ (for example, see Bishop and Bridges [4, Chapter 4, Lemma 3.7]).

To ensure the uniformity conditions for the Kan filling operator, note that we can construct piecewise linearly for each finite $A \subseteq \mathbb{N}$ and $a \in A$, dense subsets $U_{A} \subseteq[0,1]^{A}$ and retractions $\tau_{A}: D_{A} \rightarrow \bigcup_{(b, i) \in A \times 2 \backslash(a, 1)} U_{b, i}$ with the following symmetry property. Let $\pi \in \operatorname{Perm}(\mathbb{A})$ and write $\bar{\pi}$ for the function $[0,1]^{\pi A} \rightarrow[0,1]^{A}$ induced by composition. Then $D_{\pi A}=\bar{\pi}^{-1}\left(D_{A}\right)$ and $\tau_{\pi A}=\bar{\pi}^{-1} \circ \tau_{A} \circ \bar{\pi}$. If we use retractions with these symmetry conditions in the proof above we ensure the uniformity conditions are satisfied.

Remark 1.11 When we try to define a function on $[0,1]^{\mathbb{N}}$ piecewise linearly, the best we can do constructively in general is to define the function on a dense subset. This is why we work with dense subsets of $[0,1]^{\mathbb{N}}$ in the proof of Proposition 1.10 , and why we need the extra assumption of completeness of $X$. See [10], where Palmgren discusses a similar issue for a related property called the path joining property. In fact the path joining property follows from the fibrancy of the nerve of a metric space, so we cannot show the nerve of $\{x \in[-1,1] \mid x \leq 0 \vee x \geq 0\}$ is fibrant constructively. This is because by [10, Proposition 2.3] the path joining property for that space implies LLPO, the lesser limited principle of omniscience, which states that for all $\alpha: \mathbb{N} \rightarrow 2$, if $\alpha$ is 1 at most once, then either $\alpha(2 i)=0$ for all $i$, or $\alpha(2 i+1)=0$ for all $i$. (Hence also we cannot show that this space is complete without assuming LLPO).

As in [10], another approach that promises to be better behaved for more general results is to use formal topologies instead of metric spaces. In this paper all the examples we use will be complete metric spaces.

\subsection{Algebraic Weak Factorisation Systems}

Weak factorisation systems are widely used in homotopical algebra, and are defined as follows. 
(1) Let $\mathbb{C}$ be a category, and let $i: U \rightarrow V$ and $f: X \rightarrow Y$ be morphisms in $\mathbb{C}$. We say $i$ has the left lifting property with respect to $f$, and $f$ has the right lifting property with respect to $i$ and write $i \pitchfork f$ to mean that for every commutative square of the following form,

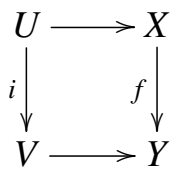

there exists a diagonal map $j: V \rightarrow X$ making two commutative triangles.

(2) Let $\mathcal{M}$ be a class of morphisms. We define the following classes.

$$
\begin{aligned}
& M^{\pitchfork}:=\{f \mid(\exists i \in \mathcal{M}) i \pitchfork f\} \\
& { }^{\pitchfork} M:=\{i \mid(\exists f \in \mathcal{M}) i \pitchfork f\}
\end{aligned}
$$

(3) For classes $\mathcal{M}$ and $\mathcal{N}$ we write $\mathcal{M} \pitchfork \mathcal{N}$ to mean that for all $i \in \mathcal{M}$ and for all $f \in \mathcal{N}$ we have $i \pitchfork f$.

(4) A weak factorisation system is two classes of maps $\mathcal{L}$ and $\mathcal{R}$ such that $\mathcal{L}={ }^{\dagger} \mathcal{R}$, $\mathcal{R}=\mathcal{L}^{\pitchfork}$ and every morphism in $\mathbb{C}$ factors as a morphism in $\mathcal{L}$ followed by a morphism in $\mathcal{R}$.

Algebraic weak factorisation systems (awfs's), originally called natural weak factorisation systems, are a variation developed by Grandis and Tholen in [8]. Garner showed in [7] that a form of the small object argument can be used to construct awfs's from a diagram of left maps. However, the proof uses transfinite arguments that may be problematic constructively. Riehl used awfs's in [15] as the main ingredient in the theory of algebraic model structures; the same paper contains a comprehensive introduction to awfs's.

We recall the definition of awfs below. We write $\mathbf{2}$ and $\mathbf{3}$ for the categories given by linear orderings with 2 elements and 3 elements respectively.

Definition 1.12 Let $\mathbb{C}$ be a category. Note that there is a canonical functor $\mathbb{C}^{3} \rightarrow \mathbb{C}^{2}$ given by composition. A functorial factorisation on $\mathbb{C}$ is a functor $\mathbb{C}^{2} \rightarrow \mathbb{C}^{\mathbf{3}}$ that is a section of the composition functor.

Throughout this paper we will write out functorial factorisations as three separate components: a functor $K: \mathbb{C}^{2} \rightarrow \mathbb{C}$ together with maps $\lambda_{f}$ and $\rho_{f}$ for each morphism 
$f: X \rightarrow Y$ in $\mathbb{C}$, as in the diagram below:

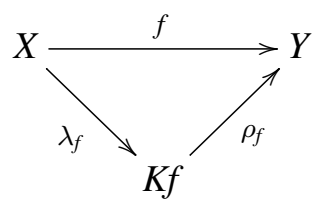

For each functorial factorisation we may define a copointed endofunctor $L: \mathbb{C}^{2} \rightarrow \mathbb{C}^{2}$, whose action on objects is given by sending $f$ to $\lambda_{f}$, and a pointed endofunctor $R: \mathbb{C}^{2} \rightarrow \mathbb{C}^{2}$, whose action on objects is given by sending $f$ to $\rho_{f}$. For full details see Grandis and Tholen [8], Garner [7] or Riehl [15].

Definition 1.13 (Grandis, Tholen) Let $\mathbb{C}$ be a category. An algebraic weak factorisation system on $\mathbb{C}$ consists of a functorial factorisation $K, \lambda, \rho$, together with natural transformations $\Sigma: L \rightarrow L^{2}$ and $\Pi: R^{2} \rightarrow R$ such that $L$ together with $\Sigma$ form a comonad, $L$ (with $\Sigma$ the comultiplication map), and $\Pi$ together with $R$ form a monad R.

Note in particular that the multiplication on $R$ gives us an $R$-algebra structure on $\rho_{f}$ for any $f$ (as is the case for any monad), and dually comultiplication gives $\lambda_{f}$ the structure of an $L$-coalgebra for every $f$.

\section{Construction of Functorial Factorisation}

\subsection{Construction of Factorisation}

We now define the awfs on 01Sub. The basic idea is the same as Garner's small object argument, as in [7], but is somewhat simpler here than in general. This can also be seen as a generalisation of Kan completion, as defined by Huber in [9, Section 3.5].

We first define the functor $K: 01 \mathrm{Sub}^{2} \rightarrow 01 \mathrm{Sub}$ that will provide the objects of the functorial factorisation $01 \mathrm{Sub}^{2} \rightarrow 01 \mathrm{Sub}^{3}$. The basic intuition here is that we know the map $\rho: K f \rightarrow Y$ should be a Kan fibration. Hence we freely add fillers for open boxes to $K f$ to ensure this is the case. We use the set of open boxes itself to do this, with the idea that each open box is its own filler. In order for $K f$ to be an object in 01Sub we need to ensure that if $(u, y)$ is a 1 -open $(A, a)$-box that has been added to $K f$, then the substitution $(u, y)(a:=1)$ is well defined. For this, we add another component $K^{+} f$ which is a subset of $[\mathbb{A}] K f$, corresponding to what Bezem, Coquand and Huber 
refer to as Kan composition in [3]. The remaining substitutions are already determined by the conditions on Kan filler operations, including the uniformity conditions so we don't need to add anything more regarding 1 open boxes. We then do the same thing for 0 -open boxes and iterate $\omega$ times.

Fix $f: X \rightarrow Y$. We will define $K f$ in $\omega$ stages. For each $n \in \omega$, we will define inductively $K_{n} f \in 01$ Sub. We will simultaneously define $\rho_{n}: K_{n} f \rightarrow Y$ and $\lambda_{n}: K_{n} f \rightarrow K_{n+1} f$. We will also ensure that $\rho_{n+1} \circ \lambda_{n}=\rho_{n}$.

If we have already defined $K_{m} f$ for $m<n$ then we define $\operatorname{colim}_{m<n} K_{m} f$ to be the colimit over $K_{m} f$ for $m<n$ together with the maps $\lambda_{m}$. We now define $K_{n} f$, assuming that $K_{m} f$ has already been defined for $m<n$. We first define nominal sets $K_{n}^{\uparrow} f, K_{n}^{+} f$, $K_{n}^{\downarrow} f$ and $K_{n}^{-} f$.

(1) Define $K_{n}^{\uparrow} f$ to be pairs $(u, y)$ where $u$ is a 1 -open box in $\operatorname{colim}_{m<n} K_{m} f$ over $y$. The action of permutations on $K_{n}^{\uparrow} f$ is defined componentwise.

(2) Define $K_{n}^{+} f$ to be the subset of $[\mathbb{A}] K_{n}^{\uparrow} f$ consisting of $\langle a\rangle(u, y)$ where $u$ is a 1 -open $A, a$-box in $\operatorname{colim}_{m<n} K_{m} f$ over $y$. More formally, we define an equivariant subset of $\mathbb{A} \times K_{n}^{\uparrow} f$ as the set of pairs $(a,(u, y))$ where $u$ is an $A, a$-box for some finite $A$ with $a \in A$, and then define $K_{n}^{+} f$ to be image of this subset under the projection $\mathbb{A} \times K_{n}^{\uparrow} f \rightarrow[\mathbb{A}] K_{n}^{\uparrow} f$.

(3) Define $K_{n}^{\downarrow} f$ analogously to $K_{n}^{\uparrow} f$ but for 0 -open boxes.

(4) Define $K_{n}^{-} f$ analogously to $K_{n}^{+} f$ but for 0 -open boxes.

We now define $K_{n} f$ to be coproduct $X \amalg K_{n}^{\uparrow} f \amalg K_{n}^{+} f \amalg K_{n}^{\downarrow} f \amalg K_{n}^{-} f$ in nominal sets. Since we have not defined a substitution structure on the individual components, it would not make sense to use the coproduct in 01Sub. We will show below how to define a substitution structure on $K_{n} f$.

Note that there is a natural injection of $K_{n-1}^{\uparrow} f$ into $K_{n}^{\uparrow} f$, and similarly for the other components of the disjoint union. Hence we can define $\lambda_{n-1}$ componentwise. We also define $\rho_{n}$ componentwise, as follows.

(1) If $x \in X$, we define $\rho_{n}(x)=f(x)$.

(2) If $(u, y) \in K_{n}^{\uparrow} f$ is a 1 -open $A, a$-box, we define $\rho_{n}(u, y)=y$.

(3) If $\langle a\rangle(u, y) \in K_{n}^{+} f$, we define $\rho_{n}(\langle a\rangle(u, y)):=y(a:=1)$. Formally, to show this is well defined, first use the above description to get a partial function on pairs $(a,(u, y))$ where $(u, y)$ is a 1 -open $A, a$ box and apply Lemma 1.2. Note that $a$ is fresh for $y(a:=1)$ by the axioms for 01 -substitution sets. 
(4) For $K_{n}^{\downarrow} f$, we define $\rho$ analogously to $K_{n}^{\uparrow} f$.

(5) For $K_{n}^{-} f$, we define $\rho$ analogously to $K_{n}^{+} f$.

Since $K_{n} f$ was defined as a coproduct in nominal sets, we already have an action of $\operatorname{Perm}(\mathbb{A})$ on $K_{n} f$ (which is just defined componentwise), but we still need to define the action of substitutions. If $x$ is in the "copy" of $X$ in $K_{n} f$, then define $x(a:=i)$ to be the same as in $X$ itself. If $(u, y)$ is an element of $K_{n}^{\uparrow} f$, with $u$ an $A, a$ box then define $(u, y)\left(a^{\prime}:=i\right)$ by cases. In the below, we write $\lambda_{m<n}$ to mean the natural injection of $\operatorname{colim}_{m<n} K_{m} f$ into $K_{n} f$.

$$
(u, y)\left(a^{\prime}:=i\right):= \begin{cases}\lambda_{m<n}\left(u\left(a^{\prime}, i\right)\right) & \text { if }\left(a^{\prime}, i\right) \in A \times 2 \backslash(a, 1) \\ \langle a\rangle(u, y) \in K_{n}^{+} f & \text { if } a^{\prime}=a \text { and } i=1 \\ \left(u\left(a^{\prime}:=i\right), y\left(a^{\prime}:=i\right)\right) \in K_{n}^{\uparrow} f & \text { otherwise }\end{cases}
$$

Substitution for $K_{n}^{\downarrow} f$ is defined similarly.

If $\langle a\rangle(u, y)$ is an element of $K_{n}^{+} f$, with $u$ a 1 -open $A, a$-box, then we define $\langle a\rangle(u, y)\left(a^{\prime}:=i\right)$ as follows. Note that by applying corollary 1.3 we may assume without loss of generality that $a^{\prime} \neq a$.

$$
\langle a\rangle(u, y)\left(a^{\prime}:=i\right):= \begin{cases}\lambda_{m<n}\left(u\left(a^{\prime}, i\right)(a:=1)\right) & \text { if } a^{\prime} \in A \\ \langle a\rangle\left(u\left(a^{\prime}:=i\right), y\left(a^{\prime}:=i\right)\right) & \text { otherwise }\end{cases}
$$

To check that we do satisfy the conditions of corollary 1.3, we note that in both cases $a$ is fresh for $\langle a\rangle(u, y)\left(a^{\prime}:=i\right)$. Substitution for $K_{n}^{-} f$ is defined similarly.

To show that $K_{n} f$ is a 01 -substitution set, we need to check the axioms for 01 -substitution operation listed in definition 1.4.

These can be checked by induction on $n$, splitting into cases depending on which disjoint component of $K_{n} f x$ lies in. For axioms 1 and 3 in the definition of 01 -substitution operation it is necessary to use the freshness and adjacency conditions respectively in the definition of open box.

We now define $K f$ to be the colimit over all $K_{n} f$ (together with the injections $\lambda_{n}$ ). We also have an obvious morphism $\lambda_{f}: X \rightarrow K f$ given by inclusion $X$ into $K_{0} f$, and a morphism $\rho_{f}: K f \rightarrow Y$ using the $\rho_{n}$.

\subsection{Rank is Well Defined}

Note that for every $n$ there is a canonical map $\lambda_{n<\omega}: K_{n} f \rightarrow K f$. In the following lemma we show that for every $x \in K f$ there is a least $n$ such that $x$ appears in the image 
of such a map. Note that constructively, we cannot simply appeal to the well ordering of $\omega$.

Lemma 2.1 For every $x \in K f$, there is a least $n$ such that there is $x^{\prime} \in K_{n} f$ such that $\lambda_{n<\omega}\left(x^{\prime}\right)=x$. Furthermore, $x^{\prime}$ is uniquely determined.

Proof First note that since decidable inhabited subsets of $\omega$ always have least elements, it suffices to show that for each $x \in K_{m} f$ and for any $n$ either there exists $x^{\prime} \in K_{n} f$ such that $\lambda_{n<\omega}\left(x^{\prime}\right)=\lambda_{m<\omega}(x)$ or there does not, and that $x^{\prime}$ is unique if it exists.

We now show the statement above by induction on $m$. Note that we may assume without loss of generality that $n<m$.

Now given $x \in K_{m} f$, note that $x$ must belong to one of the five components in the disjoint union forming $K_{m} f$.

If $x \in X$, then for all $n$ there exists a suitable $x^{\prime}$.

Now suppose that $x$ is of the form $(u, y)$ where $u$ is a 1 -open $A, a$-box. For every $\left(a^{\prime}, i\right) \in A \times 2 \backslash(a, 1)$, we have that $u\left(a^{\prime}, i\right) \in \operatorname{colim}_{m^{\prime}<m} K_{m^{\prime}} f$. Hence we know by the induction hypothesis that for each $\left(a^{\prime}, i\right) \in A \times 2 \backslash(a, 1), u\left(a^{\prime}, i\right)$ either belongs to $K_{n^{\prime}} f$ for some $n^{\prime}<n$ or does not. Since $A \times 2 \backslash(a, 1)$ is finite, we deduce that the statement that $u\left(a^{\prime}, i\right)$ belongs to the image of $\operatorname{colim}_{n^{\prime}<n} K_{n^{\prime}} f$ for every $\left(a^{\prime}, i\right) \in A \times 2 \backslash(a, 1)$ is either true or false. However, we will show that the statement is true precisely when there exists $x^{\prime} \in K_{n} f$ such that $\lambda_{n<\omega}\left(x^{\prime}\right)=\lambda_{m<\omega}(x)$. Suppose that every $u\left(a^{\prime}, i\right)$ does belong to the image of $\operatorname{colim}_{n^{\prime}<n} K_{n^{\prime}} f$, say $z_{a^{\prime}, i}^{\prime} \in \operatorname{colim}_{n^{\prime}<n} K_{n^{\prime}} f$. Then we can define $u^{\prime}$ by $u^{\prime}\left(a^{\prime}, i\right):=z_{a^{\prime}, i}^{\prime}$, which satisfies the adjacency conditions using the injectivity of the $\lambda_{i}$ giving us an open box in $\operatorname{colim}_{n^{\prime}<n} K_{n^{\prime}} f$ and so $\left(u^{\prime}, y\right) \in K_{n} f$. For each $\left(a^{\prime}, i\right)$, the image of $u^{\prime}\left(a^{\prime}, i\right)$ in $\operatorname{colim}_{m^{\prime}<m} K_{m^{\prime}} f$ is equal to $u\left(a^{\prime}, i\right)$, so we get $\lambda_{n<\omega}\left(\left(u^{\prime}, y\right)\right)=\lambda_{m<\omega}((u, y))$. For the converse, note that if there exists $x^{\prime} \in K_{n} f$ such that $\lambda_{n<\omega}\left(x^{\prime}\right)=\lambda_{m<\omega}((u, y))$, then $x^{\prime}$ has to be of the same form $\left(u^{\prime}, y\right)$, since each $\lambda_{i}$ maps each component in the disjoint union making up $K_{i} f$ into the corresponding component of $K_{i+1} f$. It easily follows that that $u\left(a^{\prime}, i\right)$ belongs to the image of $\operatorname{colim}_{n^{\prime}<n} K_{n^{\prime}} f$ for every $\left(a^{\prime}, i\right) \in A \times 2 \backslash(a, 1)$.

If $x$ belongs to one of the remaining 3 components, then the proof is similar to that of $K_{n}^{\uparrow} f$.

Finally uniqueness is clear again using the injectivity of the $\lambda_{i}$.

Definition 2.2 Given $x \in K f$, we refer to the $n$ in Lemma 2.1 as the rank of $x$. 
Remark 2.3 We will see later that the fact that rank is a well defined natural number implies that all left maps have decidable image (Lemma 6.4). Constructively, this is not the case in general for cofibrantly generated awfs's.

\subsection{Functoriality of $K$}

In section 2.1 we defined $K$ on the objects of $01 S_{u b}^{2}$. We now complete the construction of the functor by defining the action of $K$ on the morphisms of $01 \mathrm{Sub}^{2}$.

Given $f: X \rightarrow Y$ and $g: U \rightarrow V$ objects of $01 S^{2} b^{2}$, recall that a morphism from $f$ to $g$ is a commutative square



We also need to check that the following diagram commutes (in order to ensure that the overall factorisation $01 \mathrm{Sub}^{2} \rightarrow 01 \mathrm{Sub}^{3}$ is a functor).

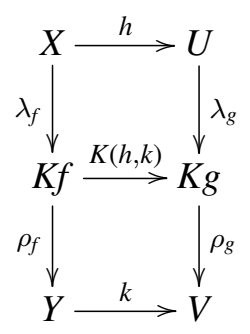

We will simultaneously check this while defining $K(h, k)$. One can also check at the same time that $K(h, k)$ is equivariant and preserves substitution, ie that it actually is a morphism in 01Sub.

We define $K(h, k): K f \rightarrow K g$ by induction on rank. If $x \in K f$ has rank 0 , then it is an element of $X$. We define $K(h, k)(x)$ to be $h(x)$. Note that this precisely ensures that the upper square of (11) commutes.

If $x$ is of the form $(u, y)$ where $u$ is a 1-open $A, a$-box, then note that for each $\left(a^{\prime}, i\right) \in A \times 2 \backslash(a, 1)$ we have that $u\left(a^{\prime}, i\right)$ is of strictly lower rank than $x$, and so we may assume that $K(h, k)\left(u\left(a^{\prime}, i\right)\right)$ has already been defined. Note that we have a 1-open $A, a$-box given by $K(h, k) \circ u$. Furthermore, note that by applying the lower square of (11) to each $u\left(a^{\prime}, i\right)$ we have that $K(h, k) \circ u$ is an open box over $k(y)$. Hence $(K(h, k) \circ u, k(y))$ is an element of $K g$, and so we can take $K(h, k)(x)$ to be 
$(K(h, k) \circ u, k(y))$. Note that we can now see that the lower square of (11) holds "locally" at $x$.

Given $\langle a\rangle(u, y) \in K^{+} f$, we define $K(h, k)$ to be $\langle a\rangle(K(h, k) \circ u, k(y))$, noting that we can use Lemma 1.2 to ensure we get a well defined function.

We can similarly define $K(h, k)$ on elements of $K^{\downarrow} f$ and $K^{-} f$.

Finally note that one can easily show by induction that $K$ preserves composition and identities.

\section{Monad and Comonad Structure}

\section{1 $L$ is a Comonad}

We define the comonad $L$ as follows. We need to define a comultiplication morphism $\Sigma: L \rightarrow L^{2}$. Given a morphism $f: X \rightarrow Y$, recall that $L f=\lambda_{f}: X \rightarrow K f$. This means that $L^{2} f$ is the morphism $\lambda_{\lambda_{f}}: X \rightarrow K \lambda_{f}$. We will define $\sigma_{f}$ such that $\Sigma_{f}$ will be the commutative square below.

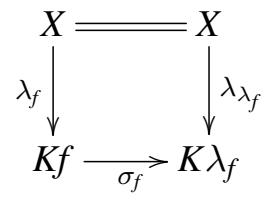

We inductively define $\sigma_{f}: K f \rightarrow K \lambda_{f}$, by taking it to be the identity on $X$ (note that this implies that (12) is commutative). Then given an element, $(u, y)$ of $K_{n}^{\uparrow} f$, send it to $\left(\sigma_{f} \circ u,(u, y)\right)$ in $K \lambda$. Given an element $\langle a\rangle(u, y)$ of $K^{+} f$, send it to $\langle a\rangle\left(\sigma_{f} \circ u,(u, y)\right)$ (which is well defined by Lemma 1.2), and similarly for the remaining components of $\left.K_{n} f\right)$. Checking that $\mathrm{L}$ is indeed a comonad amounts to checking the following three 
diagrams commute:
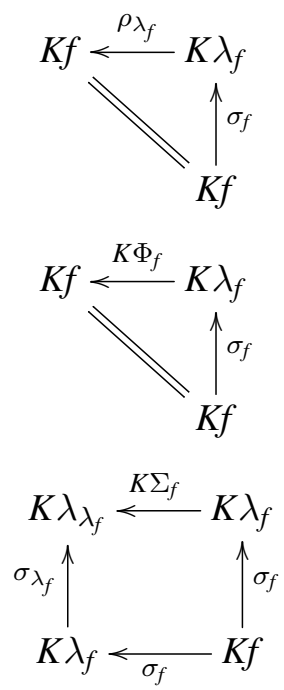

All three can be verified by induction on rank. To illustrate, we check the comultiplication law, (15) below. The case when we are given an element of $X$ is clear, so it remains to check the cases where we are given an element of the form $(u, y)$ or $\langle a\rangle(u, y)$. We will just verify the case $(u, y)$; the other case is similar.

$$
\begin{aligned}
K \Sigma_{f} \circ \sigma_{f}(u, y) & =K \Sigma_{f}\left(\sigma_{f} \circ u,(u, y)\right) \\
& =\left(K \Sigma_{f} \circ \sigma_{f} \circ u, \sigma_{f}(u, y)\right) \\
& =\left(K \Sigma_{f} \circ \sigma_{f} \circ u,\left(\sigma_{f} \circ u,(u, y)\right)\right) \\
\sigma_{\lambda_{f}} \circ \sigma_{f}(u, y) & =\sigma_{\lambda_{f}}\left(\sigma_{f} \circ u,(u, y)\right) \\
& =\left(\sigma_{\lambda_{f}} \circ \sigma_{f} \circ u,\left(\sigma_{f} \circ u,(u, y)\right)\right) \\
& =\left(K \Sigma_{f} \circ \sigma_{f} \circ u,\left(\sigma_{f} \circ u,(u, y)\right)\right) \quad \text { by induction on rank } \\
& =K \Sigma_{f} \circ \sigma_{f}(u, y)
\end{aligned}
$$

\section{2 $R$ is a Monad}

To define the monad $\mathrm{R}$, we need to define a multiplication map $\Pi: R^{2} \rightarrow R$. Given $f: X \rightarrow Y, R f$ is of the form $\rho_{f}: K f \rightarrow Y$, and so $R^{2} f$ is of the form $\rho_{\rho_{f}}: K \rho_{f} \rightarrow Y$. We will define $\pi_{f}$ so that $\Pi_{f}$ is the commutative diagram below.

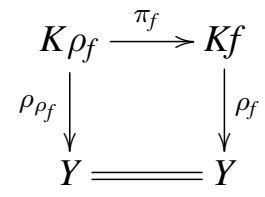


We define $\pi_{f}$ inductively. If $x \in K \rho$ is already an element of $K f$, then we can take $\pi_{f} x$ to be $x$. If $x \in K \rho_{f}$ is of the form $(u, y)$, then note that $\pi_{f} \circ u$ is an open box over $y$, and so we can take $\pi_{f} x$ to be $\left(\pi_{f} \circ u, y\right)$. To check that this is a monad, we need to verify the commutativity of the following diagrams.

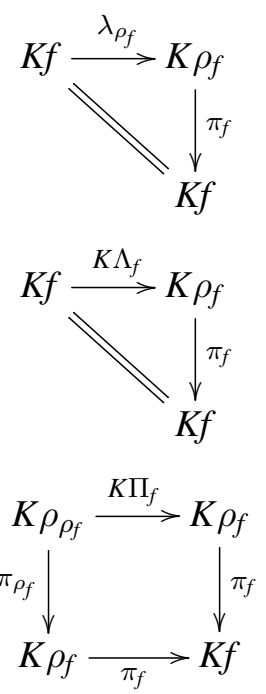

As before, each diagram can be checked by induction on rank.

\section{Kan Fibrations}

Every algebraic weak factorisation system gives rise to a weak factorisation system $(\overline{\mathcal{L}}, \overline{\mathcal{R}})$ where $\overline{\mathcal{L}}$ and $\overline{\mathcal{R}}$ are the retract closures of L-maps and R-maps respectively (the fact that this is indeed a weak factorisation system follows from standard results in homotopical algebra). In fact $\overline{\mathcal{L}}$ and $\overline{\mathcal{R}}$ can also be characterised as the class of maps that admit $L$-coalgebra and $R$-algebra structures when $L$ and $R$ are viewed as a copointed and pointed endofunctor respectively, that is, when only the counit and unit law are required and not the comultiplication and multiplication law (see Riehl [15, Lemma 2.8]). We will check that for the awfs of this paper $\overline{\mathcal{R}}=\mathcal{R}$ and show that this class is precisely the class of Kan fibrations in the sense of Pitts [12], and in fact R algebra structures correspond exactly to Kan filling operations.

Lemma 4.1 Suppose that $f: X \rightarrow Y$ is a map in 01Sub and that we are given an algebra structure for $f$ over $\mathrm{R}$ regarded as a pointed endofunctor (that is, we only require 
the unit law and not the multiplication law for algebras). Then we can produce Kan filling operators $f \uparrow$ and $f \downarrow$ for $f$ in a canonical way.

Proof A pointed endofunctor algebra structure for $f$ is precisely a map $g$ satisfying the following commutative diagrams.
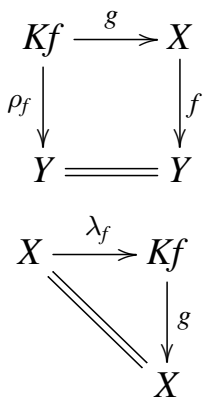

Let $(u, y)$ be an open box in $X$ over $y$ in $Y$. Note that we can regard $(u, y)$ as an element of $K f$. Define $f \uparrow(u, y)$ to be $g((u, y))$. One can easily check that this is a filler for $(u, y)$ and that $f \uparrow$ respects the uniformity conditions by applying the above two diagrams and the fact that $g$ preserves permutations and substitutions (since it is a morphism in 01Sub). Similarly for $f \downarrow$.

Lemma 4.2 Suppose that we are given $f: X \rightarrow Y$ and uniform Kan filling operations $f \uparrow$ and $f \downarrow$ for $f$. We can produce an R-algebra structure on $f$ in a canonical way.

Proof Define $g: K f \rightarrow X$ by induction. For $k$ a rank 0 element of $K f$, take $g(x)$ to be $x$. For $(u, y)$ in $K^{\uparrow} f$, note that we may assume by induction that $g \circ u$ is defined and that it is an open box over $y$ in $X$. Define $g((u, y))$ to be $f \uparrow(g \circ u, y)$. Note that this is a morphism in 01Sub (ie preserves permutations and substitutions) by applying the 
uniformity conditions. We need to check that the following diagrams commute.
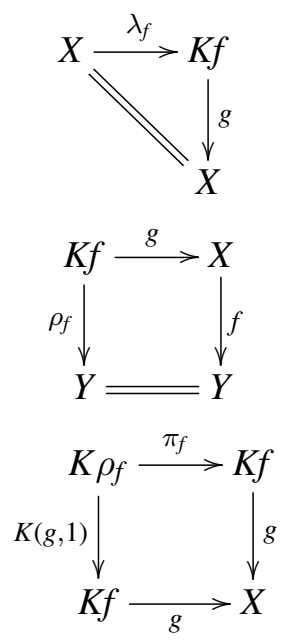

The first follows easily from the definition of $g$, and the remaining two can be shown by induction on rank.

Theorem 4.3 For $f: X \rightarrow Y$, R-algebra structures on $f$ are in precise correspondence to pairs $f \uparrow$ and $f \downarrow$ of Kan filling operators.

Proof Observe that the construction in the proof of Lemma 4.2 is a left inverse to the construction in the proof of lemma 4.1, and is also a right inverse when restricted to (monad) R-algebra structures.

Theorem 4.4 Let $f: X \rightarrow Y$ be a morphism in 01Sub. Then the following are equivalent.

(1) $f$ is an element of $\overline{\mathcal{R}}$

(2) $f$ admits a pointed endofunctor $\mathrm{R}$-algebra structure

(3) $f$ is a Kan fibration

(4) $f$ admits a (monad) R-algebra structure

Proof For $(1) \Rightarrow(2)$, this follows easily from the fact that $(\overline{\mathcal{L}}, \overline{\mathcal{R}})$ is a wfs and in particular that $\mathcal{L} \pitchfork \overline{\mathcal{R}}$. (See Riehl [15, Lemma 2.8])

For $(2) \Rightarrow(3)$, apply Lemma 4.1 .

For (3) $\Rightarrow$ (4), apply Lemma 4.2.

Finally note that $(4) \Rightarrow(1)$ is trivial. 
Remark 4.5 The implication (2) $\Rightarrow(4)$ in Theorem 4.4 is to be expected, since it holds in general for awfs's generated with Garner's version of the small object argument (see [15, Lemma 2.30]).

\section{The Generating Left Maps}

In [7], Garner shows how to define an awfs from a generating diagram of L-maps. The resulting awfs is algebraically free over the diagram. Since the construction here is a constructive variant of Garner's argument the same should be true here. In this section we define a diagram based on box inclusions and check that indeed the awfs is algebraically free over the diagram.

\subsection{Definition of the Diagram}

We now define the diagram of generating L-maps. This is based on open box inclusions for cubical sets (as described by Huber in [9, Remark 3.9]) translated into 01-substitution sets.

Let $\mathcal{J}$ be the category defined as follows. The objects of $\mathcal{J}$ are 4-tuples $(i, A, a, B)$, where $i \in 2, A$ and $B$ are finite subsets of $\mathbb{A}$ and $a \in A$. A morphism $(i, A, a, B) \rightarrow$ $\left(i^{\prime}, A^{\prime}, a^{\prime}, B^{\prime}\right)$ is a pair $(f, g)$ such that $i=i^{\prime}, f: A^{\prime} \rightarrow A$ is a bijection with $f\left(a^{\prime}\right)=a$ and $g: B^{\prime} \rightarrow B$ is a morphism in the category of names and substitutions, ie a function $B^{\prime} \rightarrow B \amalg 2$ which is "injective where defined." (Note that $f$ and $g$ are in the opposite direction to $(f, g))$.

Definition 5.1 Given a function $f: A \rightarrow \mathbb{A} \amalg 2$, say $f$ is injective where defined if the function $\left.f\right|_{f^{-1}(\mathbb{A})}$ is an injection.

Definition 5.2 Given $A$ a finite subset of $\mathbb{A}$, define the standard $A$-cube, $\square_{A}$, to be the set of functions $A \rightarrow \mathbb{A} \amalg 2$ that are injective where defined. We make $\square_{A}$ a 01 -substitution set by defining

$$
(\pi . f)(a):= \begin{cases}\pi(f(a)) & f(a) \in \mathbb{A} \\ f(a) & f(a) \in 2\end{cases}
$$

and

$$
\left(f\left(a^{\prime}:=i\right)\right)(a):= \begin{cases}f(a) & f(a) \in 2 \\ f(a) & f(a) \in \mathbb{A} \text { and } f(a) \neq a^{\prime} \\ i & f(a)=a^{\prime}\end{cases}
$$


Note that this gives a functor from the opposite of the category of names and substitutions to 01Sub. Given $f: B \rightarrow A$, define $\square_{f}: \square_{A} \rightarrow \square_{B}$ by sending $g \in \square_{A}$ to $g \circ f$. (This corresponds to the functor to cubical sets given by the Yoneda embedding)

Definition 5.3 Given $A$ a finite subset of $\mathbb{A}$ and $a \in A$, define the standard 1-open $A, a$-box, $\sqcup_{A, a}$ in 01Sub to be the subset of $\square_{A}$ consisting of $f$ such that for some $\left(a^{\prime}, i\right) \in A \times 2 \backslash\{(a, 1)\}, f\left(a^{\prime}\right)=i$. Note that this is a subobject of $\square_{A}$ by inclusion, in 01Sub.

The standard 0-open A, a-box, $\sqcap_{A, a}$, is defined analogously.

Note that the morphism $\square_{f}: \square_{A} \rightarrow \square_{B}$ given above restricts to a morphism $\sqcup_{A, a} \rightarrow$ $\sqcup_{B, b}$ if $f(b)=a$.

We define a functor $J: \mathcal{J} \rightarrow 01 \mathrm{Sub}^{2}$ as follows. On an object $(i, A, a, B)$, define $J(i, A, a, B)$ to be the map from $\sqcup_{A, a} * \square_{B}$ to $\square_{A} * \square_{B}$ defined by taking the product of the inclusion $\sqcup_{A, a} \hookrightarrow \square_{A}$ with the identity on $\square_{B}$.

Given a morphism $(f, g):(i, A, a, B) \rightarrow\left(i, A^{\prime}, a^{\prime}, B^{\prime}\right)$, define $J(f, g)$ componentwise by composing $f$ and $g$ with elements of $\square_{A}$ and $\square_{B}$ respectively.

\subsection{Algebraic Freeness}

We will show that the awfs is algebraically free on $J$. This means we need to find $\eta: \mathcal{J} \rightarrow$ L-Map over 01 Sub $^{2}$ such that the following is an isomorphism of categories (see Garner [7, Definition 3.9]).

$$
\text { R-Map } \stackrel{\text { lift }}{\longrightarrow} \text { L-Map } \stackrel{\eta^{\dagger}}{\longrightarrow} \mathcal{J}^{\pitchfork}
$$

We require that $\eta(i, A, a, B)$ is an $\mathrm{L}$-coalgebra structure on $J(i, A, a, B)$. We just show the case for 1 -open boxes. The case for 0 -open boxes is similar. We now fix $(1, A, a, B)$ and refer to $J(1, A, a, B)$ as $\iota$. To find $\eta(1, A, a, B)$ we need to find $h: \square_{A} * \square_{B} \rightarrow K \iota$ 
satisfying the following commutative diagrams.
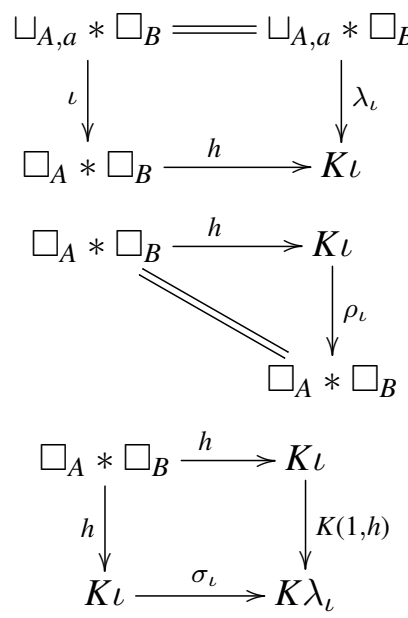

If $(f, g) \in \square_{A} * \square_{B}$ is already an element of $\sqcup_{A, a} * \square_{B}$, then define $h(f, g)$ to be $(f, g)$. Note that this precisely ensures that (19) commutes.

If $f$ is defined everywhere on $A$, then let $u$ be the 1-open $f(A), f(a)$-box defined for $a^{\prime \prime} \in f(A)$ by

$$
u\left(a^{\prime \prime}, i\right):=f\left(a^{\prime \prime}:=i\right)
$$

We then define

$$
h(f, g):=(u,(f, g))
$$

If $f$ is defined everywhere on $A \backslash a$ and $f(a)=1$, then let $b$ be a fresh variable, define $f^{\prime}$ as follows

$$
f^{\prime}\left(a^{\prime}\right):= \begin{cases}f\left(a^{\prime}\right) & a^{\prime} \in A \backslash a \\ b & a^{\prime}=a\end{cases}
$$

Then define $u^{\prime}$ as for $u$ in (22), but with $f^{\prime}$ in place of $f$, and we can now define $h(f, g) \in K^{+} \iota$ to be

$$
h(f, g):=\langle b\rangle\left(u^{\prime},\left(f^{\prime}, g\right)\right)
$$

This completes the definition of $\eta(1, A, a, B)=h$. The commutativity of (20) is clear by definition and we check (21) as follows. Since the image of $u$ in (22) is a subset of the $X$ component of $K \iota$, we have $\sigma_{\iota} \circ u=u$. Hence we deduce:

$$
\begin{aligned}
\sigma_{\iota} \circ h(f, g) & =\left(\sigma_{\iota} \circ u, h(f, g)\right) \\
& =(u, h(f, g)) \\
& =K(1, h)(u,(f, g))
\end{aligned}
$$


The case for 0 -open boxes is similar. We now show that (18) is an isomorphism by exhibiting an inverse.

The elements of $\mathcal{J}^{\pitchfork}$ are of the form $(g, \phi)$ where $g: X \rightarrow Y$ is a morphism in 01Sub and $\phi$ is lifting data for $g$ against $J$. We will use $\phi$ to define Kan filling operators on $g$ and apply Lemma 4.1 to get an R-algebra structure on $g$.

Let $(u, y)$ be an open box in $X$ over $y$ in $Y$. Let $u$ be a 1-open $A, a$-box, and let $C$ be a finite support for $u$. Let $B:=C \backslash A$, and note that $B$ is a finite set. For any $\left(a^{\prime}, i\right) \in A \times 2 \backslash(1, a)$, we can define $\overline{\left(a^{\prime}, i\right)} \in \sqcup_{A, a}$ by

$$
\overline{\left(a^{\prime}, i\right)}\left(a^{\prime \prime}\right):= \begin{cases}i & a^{\prime \prime}=a^{\prime} \\ a^{\prime \prime} & a^{\prime \prime} \neq a^{\prime}\end{cases}
$$

Now note that there is a unique morphism $\tilde{u}: \sqcup_{A, a} \rightarrow X$ such that for each $\left(a^{\prime}, i\right) \in$ $A \times 2 \backslash(a, 1), \tilde{u}\left(\overline{\left(a^{\prime}, i\right)}\right)=u\left(a^{\prime}, i\right)$. Also note that there is a unique morphism $\tilde{y}: \square_{A} * \square_{B} \rightarrow Y$ such that $\tilde{y}\left(1_{A}, 1_{B}\right)=y$. (Both of these results can be viewed as translating the Yoneda lemma from cubical sets to 01-substitution sets). These maps together make a commutative square:

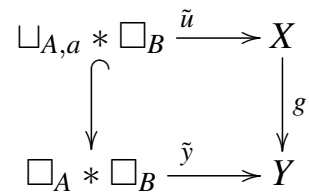

Then, applying the lifting data $\phi$ yields a diagonal filler $j: \square_{A} * \square_{B} \rightarrow X$. One can check that $j\left(1_{A}, 1_{B}\right)$ is a filler for $(u, y)$.

To show that this gives a Kan filling operation, we first need to check that it is well defined. So let $C^{\prime}$ be another finite support for $u$. Since the intersection of two finite supports is also a finite support, we may assume without loss of generality that $C^{\prime} \subseteq C$. Hence also $B^{\prime} \subseteq B$ and the inclusion $i: B^{\prime} \hookrightarrow B$ induces a morphism $(1, A, a, B) \rightarrow\left(1, A, a, B^{\prime}\right)$ in $\mathcal{J}$. Applying the coherence condition for $\phi$ to $i$, then shows that the fillers we get using $B$ and using $B^{\prime}$ are equal.

Similar arguments show that the filling operation we get satisfies the uniformity conditions.

Exactly the same argument allows us to construct a filling operator for 0-open boxes. Applying Lemma 4.1 then gives us an R-algebra structure on $g$.

Finally, one can check that this is in fact an inverse to the map (18). 
Remark 5.4 We can use this characterisation to verify that for any map $f$ in the category of cubical sets, the fibration structures on $f$ correspond precisely to the fibration structures on the image of $f$ in 01Sub under Pitts' equivalence in [13]. This is because Huber gave in [9, Remark 3.9] a characterisation of fibrations that can be easily seen as showing the fibrations are algebraically cofibrantly generated. Since it can be easily checked that the generating diagram in this section is (naturally isomorphic to) the image of the generating diagram of left maps in [9, Remark 3.9], we deduce that the resulting fibration structures are also the same (up to isomorphism).

\section{Path Objects}

As shown by Awodey and Warren in [1], identity types in type theory can be implemented using path objects, which are defined as follows.

Definition 6.1 Let $(\mathcal{L}, \mathcal{R})$ be a wfs and let $f: X \rightarrow Y$ be a map in $\mathcal{R}$. A path object on $f$ is a factorisation of the the diagonal map $\Delta: X \rightarrow X \times_{Y} X$ as a map in $\mathcal{L}$ followed by a map in $\mathcal{R}$.

Note that we can trivially generate path objects using the awfs structure itself. For any fibration $f: X \rightarrow Y$, if $\Delta: X \rightarrow X \times_{Y} X$ is the diagonal map, then $K \Delta$ is a path object. However, in order to implement identity types it is necessary for the path objects to be stable under pullback (see the statement of [1, Theorem 3.1]).

This is not the case for path objects generated using the awfs, as we prove below. This is often the case for awfs's, as remarked by van den Berg and Garner in [2, Remark 3.3.4]. The basic idea in this case is that given a continuous function $f: X \rightarrow Y$, for each point $x \in X$ and each path $p$ in $Y$ with $p(0)=f(x)$, we freely added a path $\tilde{p}$ in $X$ over $p$ with $\tilde{p}(0)=x$. However, our construction also adds the other endpoint of $\tilde{p}, \tilde{p}(1)$. In our definition of the awfs, this can be seen explicitly as elements of the $K^{+} f$ component (and similarly the $K^{-}$component). The added endpoint $\tilde{p}(1)$ contains "data" about the whole path $p$, but lies entirely in the fibre of $p(1)$. Hence the pullback along the map $1 \rightarrow Y$ given by $p(1)$ does not preserve the awfs.

Theorem 6.2 There are fibrant objects $X, Y$ and $Z$, a fibration $f: X \rightarrow Y$ and a map $g: Z \rightarrow Y$ such that, writing $g^{*}(f)$ for the pullback of $f$ along $g$, there is no map 
$K \Delta_{g^{*}(f)} \rightarrow K \Delta_{f}$ making the following diagram a pullback.

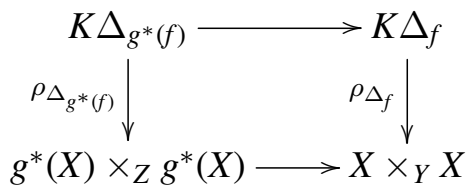

Proof Let $X=Y=N([0,1])$ (in fact any non trivial complete metric space will do), let $f$ be the identity on $N([0,1])$, and let $Z:=1$ and $g:=\lambda x .0$.

In particular, we have $X \times_{Y} X=X \times_{X} X \cong X$ and $g^{*}(X) \times_{Z} g^{*}(X) \cong 1$, so by functoriality of $K$, it suffices to show that $K 1_{1}$ (by $1_{1}$ we mean the identity on the terminal object) is not isomorphic to $1 \times_{X} K 1_{X}$.

$1 \times_{X} K 1_{X}$ contains an uncountable subset $U$, consisting of elements of the form $(*,\langle a\rangle(u, y))$ where $*$ is the only element of $1, a \in \mathbb{A}, u$ is a 1 -open $(\{a\}, a)$-box and $y \in N([0,1])$ is such that $y(a:=0)=u(a, 0)$ and $y(a:=1)=0$. To show $(*,\langle a\rangle(u, y))$ belongs to $1 \times_{X} K 1_{X}$, we need that $\rho_{f}(\langle a\rangle(u, y))=0$, but this is the case because $\rho_{f}(\langle a\rangle(u, y))=y(a:=1)=0$. Also note that in general, for any nominal sets, if $\langle a\rangle x=\langle a\rangle x^{\prime}$ then $x=x^{\prime}$.

However $K 1_{1}$ is countable, since it is a countable union of countable sets.

(For this argument to work constructively, note that $U$ is an inhabited decidable subset of $1 \times_{X} K 1_{X}$ and such subsets of countable sets are countable. Furthermore, one can find explicit enumerations of $K_{n} 1_{1}$ and thereby also an explicit enumeration of $K 1_{1}$. Then, assuming countable choice, we can use Cantor's diagonal argument to show $U$ is uncountable.)

\subsection{Name Abstraction}

The cubical sets used in Bezem, Coquand and Huber [3] to implement identity types correspond in 01-substitution sets to name abstraction.

Definition 6.3 Given $f: X \rightarrow Y$, define the name abstraction over $f,[\mathbb{A}]_{f} X$ as follows:

$$
[\mathbb{A}]_{f} X:=\{\langle a\rangle x \in[\mathbb{A}] X \mid a \# f(x)\}
$$

One can adapt the proof in [3] that identity types are Kan fibrations to show that the projection map $[\mathbb{A}]_{f} X \rightarrow X \times_{Y} X$ is an R-map. 
In order to show that $[\mathbb{A}]_{f} X$ is a path object on $X$ over $f$, it remains only to show that the reflexivity map $r: X \rightarrow[\mathbb{A}]_{f} X$ has the left lifting property with respect to every R-map. $(r(x)$ is defined to be Fresh $a$ in $\langle a\rangle x$.)

We will see later (Corollary 6.14) that assuming classical logic this is a path object. However, we show now that this cannot be done constructively. In particular, we will show that one cannot show constructively that $r: X \rightarrow[\mathbb{A}]_{f} X$ is always a left map.

Lemma 6.4 Suppose that $i: U \rightarrow V$ has the left lifting property with respect to every $\mathrm{R}$-map. Then $i$ has decidable image. (That is, every element of $V$ either lies in the image of $i$ or does not lie in the image of $i$ ).

Proof First, note that we can show that the map $\lambda_{i}: U \rightarrow K i$ has decidable image. This is because rank is well defined by Lemma 2.1, but the image of $\lambda_{i}$ is precisely the subset of $K i$ of rank 0 , and every natural number is either equal to 0 or greater than 0 .

Now by assumption, $i: U \rightarrow V$ has the left lifting property with respect to the R-map $\rho_{r}$, giving us a diagonal filler, $j$, in the following diagram:

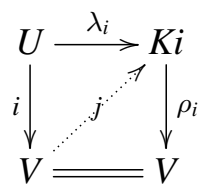

However, note that it easily follows from this diagram that any $v \in V$ lies in the image of $i$ if and only if $j(v)$ lies in the image of $\lambda_{i}$. Since we have checked that $\lambda_{i}$ has decidable image, it follows that $i$ must also have decidable image.

Theorem 6.5 It cannot be proved constructively that for every fibrant 01-substitution set, $X$, the reflexivity morphism $r: X \rightarrow[\mathbb{A}] X$ has the left lifting property with respect to every R-map.

Proof We will show this by assuming $r$ does have this property and deriving the weak limited principle of omniscience (WLPO), that is, for any $f: \mathbb{N} \rightarrow 2$ the statement " $f(n)=0$ for all $n$ " is either true or false. (In particular this implies the existence of noncomputable functions, and so is not provable constructively, even if we assume dependent choice, eg by considering the realizability model in Rathjen [14])

Let $f: \mathbb{N} \rightarrow 2$. Define $X:=N([0,1])$, the nerve of $[0,1]$. Since $[0,1]$ is complete, we have that $N([0,1])$ is fibrant by Proposition 1.10 . 
We construct a continuous function $\bar{f}:[0,1] \rightarrow[0,1]$. Given $x \in[0,1]$ we will construct a Cauchy sequence $\left(y_{n}\right)_{n<\omega}$. Let $n<\omega$. If $f(m)=0$ for all $m<n$, then let $y_{n}:=0$. If there is $m<n$ such that $f(m)=1$, then there is a least $m_{0}$ such that $f\left(m_{0}\right)=1$. Let $y_{n}:=2^{-m_{0}} q$, where $q$ is chosen such that $|q-x|<2^{-n}$. Note that in classical logic $\bar{f}$ could have been defined

$$
\bar{f}(x)= \begin{cases}0 & f(n)=0 \text { for all } n \\ 2^{-n} x & \text { there is } n \text { least such that } f(n)=1\end{cases}
$$

Now note that we can view $\bar{f}$ as an element of $X$ dependent on (at most) a single name, say, $a$ by Lemma 1.9. So we have that $\langle a\rangle \bar{f} \in[\mathbb{A}] X$.

We then have that $\langle a\rangle \bar{f}$ lies in the image of $r$ if and only if $a$ is fresh for $\bar{f}$, which is the case precisely when $\bar{f}$ is constant with respect to $a$, which in turn happens precisely when $f$ is constantly 0. But we showed in Lemma 6.4 that the image of $r$ must be decidable. Therefore, WLPO follows as required.

\subsection{Labelled Name Abstraction}

In this section we give a new construction of path object that is valid constructively and preserved by pullback. The basic idea is to add "labels" to the "name abstraction" identity types. Any side of a cube that has been "labelled" in guaranteed to be degenerate (although there may be additional degenerate sides that are not labelled). In order to make the notation and proofs slightly easier we use some notions from the construction of the awfs. In particular, we have already checked that $K \Delta$ is a 01-substitution set, so we know, for instance that "commutativity of substitution" holds, ie $z(a:=i)\left(a^{\prime}:=i^{\prime}\right)=z\left(a^{\prime}:=i^{\prime}\right)(a:=i)$. A full proof that these objects can be used to model identity types will be left for another paper, using ideas from algebraic model structures. For now we simply give a definition and verify that they are path objects and stable under pullback.

Definition 6.6 Let $f: X \rightarrow Y$ be a fibration and let $a \in \mathbb{A}$. Write $\Delta$ for the diagonal map $X \rightarrow X \times_{Y} X$ and define the subset $P_{Y}^{0} X$ of $K \Delta$ of pre-normal forms in direction $a$ inductively as follows.

(1) If $x \in X$ and $a \# x$ then $x$ is a pre-normal form in direction $a$.

(2) If $u$ is a 1 -open $A, a$-box over $\left(x_{1}, x_{2}\right), a \# x_{1}, u(a, 0)=x_{1}$ and for every $a^{\prime}, i \in(A \backslash a) \times 2, u\left(a^{\prime}, i\right)$ is a pre-normal form in direction $a$, then $\left(u,\left(x_{1}, x_{2}\right)\right)$ is a pre-normal form in direction $a$. (Note that since $\left(x_{1}, x_{2}\right)$ belongs to $X \times_{Y} X$ by the definition of $K \Delta$ we also have automatically that $f\left(x_{1}\right)=f\left(x_{2}\right)$.) 
Note that $P_{Y}^{0} X$ is closed under permutations, and every substitution of the form $\left(a^{\prime}:=i\right)$ where $a^{\prime} \neq a$.

An element of $K \Delta$ is a normal form if it is equal to $z(a:=1)$ where $z$ is some pre-normal form in direction $a$ for some $a \in \mathbb{A}$. (Note that if $a^{\prime}$ is fresh for $z$ and $a$, then the normal form can also be written as $\left(\left(a^{\prime} a\right) \cdot z\right)\left(a^{\prime}:=1\right)$ where $\left(a^{\prime} a\right) \cdot z$ is also a pre-normal form, so this is independent of the choice of $a$ ).

We refer to the set of normal forms as $P_{Y} X$. Note that it is closed under permutations and substitutions, and so we can view it as a 01-substitution set in the natural way.

Lemma 6.7 Let $z, z^{\prime}$ be pre-normal forms in direction $a$. If $z(a:=1)=z^{\prime}(a:=1)$ then $z=z^{\prime}$.

Proof Note that if $z$ is an element of $X$ with $a \# z$, then $z(a:=1)=z$. If $z$ is an open box in direction $a$, then $z(a:=1)$ is an element of $K^{+} \Delta$.

Therefore, if $z(a:=1)=z^{\prime}(a:=1)$, then either $z$ and $z^{\prime}$ are both elements of $X$, or they are both elements of $K^{\uparrow} \Delta$.

In the former case, we have $z(a:=1)=z$ and $z^{\prime}(a:=1)=z^{\prime}$ (since $a \# z$ and $\left.a \# z^{\prime}\right)$ and so $z=z^{\prime}$ as required.

In the latter case, we have that $z$ and $z^{\prime}$ are of the form $\left(u,\left(x_{1}, x_{2}\right)\right)$ and $\left(u^{\prime},\left(x_{1}^{\prime}, x_{2}^{\prime}\right)\right)$ respectively. Then $z(a:=1)$ and $z^{\prime}(a:=1)$ are the elements of $K^{+} \Delta,\langle a\rangle\left(u,\left(x_{1}, x_{2}\right)\right)$ and $\langle a\rangle\left(u^{\prime},\left(x_{1}^{\prime}, x_{2}^{\prime}\right)\right)$. Since $\langle a\rangle\left(u,\left(x_{1}, x_{2}\right)\right)=\langle a\rangle\left(u^{\prime},\left(x_{1}^{\prime}, x_{2}^{\prime}\right)\right)$, we have $\left(u,\left(x_{1}, x_{2}\right)\right)=$ $\left(u^{\prime},\left(x_{1}^{\prime}, x_{2}^{\prime}\right)\right)$ as required.

Corollary 6.8 For every normal form $w$ and every $a \in \mathbb{A}$ fresh, there is a unique pre-normal form $z$ in direction $a$ such that $z(a:=1)=w$.

Unlike the path objects from the awfs, this construction is stable under pullback, as we show below.

Theorem 6.9 Let $f: X \rightarrow Y$ and suppose we are given a commutative square of the form below.

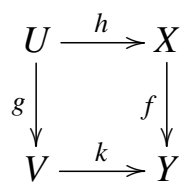


Then we can define a map $P_{V} U \rightarrow P_{Y} X$ making the following square commute.

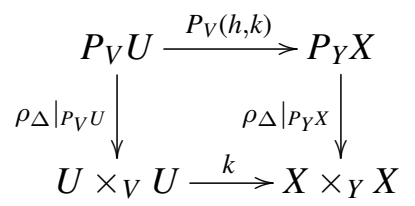

Furthermore, if (30) is a pullback square, then so is (31).

Proof The morphisms $P_{V} U \rightarrow P_{Y} X$ can easily be defined by using corollary 6.8 to lift to $P^{0}$, and then working by induction on the construction of $P^{0}$. Explicitly, given an element $u$ in the $U$ component of $P_{V}^{0} U$, we send it to $h(u)$. Given an open box $v$ over $u_{1}, u_{2}$, we send it to an open box $P_{V}^{0}(h, k) \circ v$ over $h\left(u_{1}\right), h\left(u_{2}\right)$. This definition clearly makes (31) commute.

Now assume that (30) is a pullback. Note that to show (31) is a pullback, it suffices to show that the square below is a pullback, where we write $p_{f}$ for the map $P_{Y} X \rightarrow Y$ given by composition of the restriction of $\rho_{\Delta}$ to $P_{Y} X$ with the canonical map $X \times_{Y} X \rightarrow Y$, and similarly $p_{g}$ for the analogous map $P_{V} U \rightarrow V$.

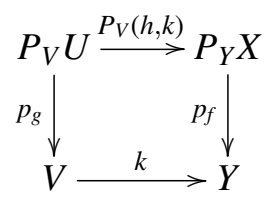

Now to check that this square is a pullback, it suffices to show that the canonical map $P_{V} U \rightarrow V \times{ }_{Y} P_{Y} X$ is an isomorphism. So we need to show that given $(v, x) \in V \times{ }_{Y} P_{Y} X$, there is a unique $u \in P_{V} U$ mapped to $(v, x)$. This is done by induction on the construction of $P^{0}$. If $x \in X$, then we can use the fact that (30) is a pullback. We now deal with the case where $x$ is of the form $\langle a\rangle\left(w,\left(x_{1}, x_{2}\right)\right)$. The key point here is that we required in Definition 6.6 that $a \# x_{1}$. Hence, writing $\pi$ for the canonical map $X \times_{Y} X \rightarrow Y$, we can deduce

$$
\begin{aligned}
p_{f}\left(\langle a\rangle\left(w,\left(x_{1}, x_{2}\right)\right)\right) & =\pi\left(\rho_{\Delta}\left(\langle a\rangle\left(w,\left(x_{1}, x_{2}\right)\right)\right)\right) \\
& =\pi\left(x_{1}(a:=1), x_{2}(a:=1)\right) \\
& =f\left(x_{1}(a:=1)\right) \\
& =f\left(x_{1}\right)
\end{aligned}
$$$$
\text { (since } \left.a \# x_{1}\right)
$$

Since $(v, x) \in V \times{ }_{Y} P_{Y} X$ we must also have $p_{f}\left(\left(w,\left(x_{1}, x_{2}\right)\right)\right)=k(v)$, and so $k(v)=$ $f\left(x_{1}\right)=f\left(x_{2}\right)$. But now using that (30) is a pullback, we have uniquely specified $u_{1}, u_{2}$ 
in $U$ such that $g\left(u_{1}\right)=g\left(u_{2}\right)=v, h\left(u_{1}\right)=x_{1}$ and $h\left(u_{2}\right)=x_{2}$. Applying the inductive hypothesis, we also have a uniquely determined open box over $w$, so there is a unique element of $P_{V} U$ mapped to $\langle a\rangle\left(w,\left(x_{1}, x_{2}\right)\right)$, as required.

We now show that this construction does give us path objects.

Theorem 6.10 The restriction of $\rho_{\Delta}: K \Delta \rightarrow X \times_{Y} X$ to $P_{Y} X$ is a fibration.

Proof Let $v$ be a 1 -open $A, a$-box in $P_{Y} X$ over $\left(x_{1}, x_{2}\right)$. Let $b$ be a fresh variable. By applying corollary 6.8 we get a 1-open $A, a$-box $v^{\prime}$ over $P_{Y}^{0} X$ satisfying the adjacency conditions by Lemma 6.7 and such that for each $\left(a^{\prime}, i\right) \in A \times 2 \backslash(a, 1), v^{\prime}\left(a^{\prime}, i\right)$ is a pre-normal form in direction $b$. Note that $\pi_{2} \circ \rho_{\Delta} \circ v^{\prime}$ is an open box in $X$ (where $\pi_{2}$ is the second projection). Let $A^{\prime}$ be $A \cup\{b\}$. We extend $\pi_{2} \circ \rho_{\Delta} \circ v^{\prime}$ to a 1 -open $A^{\prime}, a$-box $v^{\prime \prime}$ by setting $v^{\prime \prime}(b, 0)=x_{1}$ and $v^{\prime \prime}(b, 1)=x_{2}$.

Let $A^{\prime \prime}$ be $A^{\prime} \backslash a$. We define a 1 -open $A^{\prime \prime}, b$-box, $w$ as follows. Define $w(b, 0)$ to be $x_{1}(a:=1)$. For $\left(a^{\prime}, i\right) \in A^{\prime} \times 2$, define $w\left(a^{\prime}, i\right)$ to be $v^{\prime}\left(a^{\prime}, i\right)(a:=1)$. Note that $\left(w,\left(x_{1}(a:=1), f^{+} v^{\prime \prime}\right)\right)$ is a pre-normal form.

We now form a 1 -open $A^{\prime}, b$-box, $w^{\prime}$ as follows. Set $w^{\prime}(b, 0)$ to be $x_{1}$. Set $w^{\prime}(a, 1)$ to be $\left(w,\left(x_{1}(a:=1), f^{+} v^{\prime \prime}\right)\right)$. For $\left(a^{\prime}, i\right) \in A \times 2 \backslash(a, 1)$, define $w^{\prime}\left(a^{\prime}, i\right)$ to be $v^{\prime}\left(a^{\prime}, i\right)$.

Finally this allows to define the Kan filler of $v$ to be

$$
\left(w^{\prime},\left(x_{1}, f \uparrow v^{\prime \prime}\right)\right)(b:=1)
$$

We now show that $r$ is a left map. The key point in the lemmas below is to ensure that we always treat elements of the $X$ component in $P_{Y}^{0} X$ as degenerate paths, while also ensuring substitutions are preserved.

Lemma 6.11 There is a nominal set morphism $h: P_{Y}^{0} X * \mathbb{A} \rightarrow X$ such that given $z$ in direction $a$, with $b$ a fresh name, and $a^{\prime}$ a name with $a^{\prime} \neq a$ and $a^{\prime} \neq b$, we have $h\left(z\left(a^{\prime}:=i\right), b\right)=h(z, b)\left(a^{\prime}:=i\right),(h(z, b)(b:=0), h(z, b)(b:=1))=\rho_{\Delta} z$, and for $x \in X, h(x, b)=x$.

Proof We define $h$ by induction on the construction of $P_{Y}^{0} X$. We define $h(x, b)$ to be $x$. Suppose we are given a pre-normal form of the form $\left(u,\left(x_{1}, x_{2}\right)\right)$, where $u$ is a 1 -open $A, a$-box and $b$ a fresh name. Let $A^{\prime}:=A \cup\{b\}$. We form a 1-open $A^{\prime}, a$-box, $v$ in $X$ as follows. If $\left(a^{\prime}, i\right)$ is an element of $A \times 2 \backslash(a, 1)$, then we may assume by induction that $h\left(u\left(a^{\prime}, i\right), b\right)$ has already been defined. Let $v\left(a^{\prime}, i\right)$ be $h\left(u\left(a^{\prime}, i\right), b\right)$. Let $v(b, 0)$ be $x_{1}$ and let $v(b, 1)$ be $x_{2}$. Now define $h\left(\left(u,\left(x_{1}, x_{2}\right)\right), b\right)$ to be $f \uparrow v$. 
To illustrate $h$, consider when $x_{2}$ is a path with $x_{1}$ as one of the endpoints. In this case, $h$ is a homotopy from the constant path at $x_{1}$ to the path $x_{2}$. If we are given an element $x$ of the $X$ component of $P_{Y}^{0} X$, then we think of it as the degenerate path from $x$ to itself, and then return the degenerate homotopy from an endpoint of the degenerate path to the path (and in this case all of the objects mentioned happen to be equal as elements of $X$ ).

Next, in the lemma below we again produce a homotopy from a path to its endpoint, but this time viewing paths as elements of $P_{Y}^{0} X$ rather than elements of $X$, so we have some extra structure to take care of.

Lemma 6.12 There is a nominal set morphism $k: P_{Y}^{0} X * \mathbb{A} \rightarrow P_{Y}^{0} X$ such that if $a$ is the direction of $z \in P_{Y}^{0} X, b$ is a fresh name and $a^{\prime}$ is a name with $a^{\prime} \neq a$ and $a^{\prime} \neq b$, then $k\left(z\left(a^{\prime}:=i\right), b\right)=k(z, b)\left(a^{\prime}:=i\right)$, and for $x \in X$ we have $k(x, b)=x$ and such that if $z \in P_{Y}^{0} X$ and $\rho_{\Delta}(z)=\left(x_{1}, x_{2}\right)$, then $k(z, b)(b:=0)=x_{1}$ and $k(z, b)(b:=1)=z . k$ will also be "direction preserving."

Proof We define $k(x, b)$ to be $x$ for $x \in X$. Now suppose we are given a pre-normal form of the form $\left(u,\left(x_{1}, x_{2}\right)\right)$, where $u$ is a 1 -open $A, a$-box and $b$ a fresh name. Let $A^{\prime}:=A \cup\{b\}$ as before. We form a 1 -open $A^{\prime}, a$ box $v$. Given $\left(a^{\prime}, i\right) \in A \times 2 \backslash(a, 1)$, set $v\left(a^{\prime}, i\right)$ to be $k\left(u\left(a^{\prime}, i\right), b\right)$. Set $v(b, 0)$ to be $x_{1}$, and set $v(b, 1)$ to be $\left(u,\left(x_{1}, x_{2}\right)\right)$. Finally, define $k\left(\left(u,\left(x_{1}, x_{2}\right)\right), b\right)$ to be $\left(v,\left(x_{1}, h\left(\left(u,\left(x_{1}, x_{2}\right)\right), b\right)\right)\right)$, where $h$ is as in Lemma 6.11 .

Theorem 6.13 The inclusion $r: X \rightarrow P_{Y} X$ is an element of $\overline{\mathcal{L}}$.

Proof We need to define a copointed endofunctor coalgebra on $r$.

We first define a morphism $l: P_{Y}^{0} X * \mathbb{A} \rightarrow K r$, ensuring that if $z \in P_{Y}^{0} X$ is in direction $a$, then $a$ is fresh for $l(z, b)$. As before, we ensure that $l$ is a nominal set morphism that preserves substitutions $\left(a^{\prime}:=i\right)$ for $a^{\prime} \neq a, b$. We will also ensure that $\rho_{r}(l(z, b))=k(z, b)(a:=1)$.

Define $l(x, b)$ to be $x$. Given $\left(u,\left(x_{1}, x_{2}\right)\right)$ and $b$, where $u$ is an $A, a$-box, let $A^{\prime}:=$ $(A \backslash a) \cup\{b\}$. We define a 1 -open $A^{\prime}, b$-box, $v$ as follows. Given $\left(a^{\prime}, i\right) \in(A \backslash a) \times 2$, define $v\left(a^{\prime}, i\right)$ to be $l\left(u\left(a^{\prime}, i\right), b\right)$. Define $v(b, 0)$ to be $x_{1}$. Then define $l\left(\left(u,\left(x_{1}, x_{2}\right)\right), b\right)$ to be $\left(v, k\left(\left(u,\left(x_{1}, x_{2}\right)\right), b\right)(a:=1)\right)$, where $k$ is as in Lemma 6.12 .

This now allows us to define the coalgebra map $c: P_{Y} X \rightarrow K r$ by defining $c(z(a:=1))$ (for $z$ a prenormal form in direction $a$ ) to be $l(z, b)(b:=1)$, where $b$ is any fresh variable (so $c\left(\left(u,\left(x_{1}, x_{2}\right)\right)(a:=1)\right)$ will be an element of $\left.K^{+} r\right)$. Note that we have 
ensured throughout that given an element $x$ of $X, c(x)=x$. Finally to show that this is a coalgebra structure we verify the counit law below.

$$
\begin{aligned}
\rho_{r}(c(z(a:=1))) & =\rho_{r}(l(z, b)(b:=1)) \\
& =\rho_{r}(l(z, b))(b:=1) \\
& =k(z, b)(a:=1)(b:=1) \\
& =k(z, b)(b:=1)(a:=1) \\
& =z(a:=1)
\end{aligned}
$$

Corollary 6.14 Assume that every $x \in X$, has a (necessarily unique) least finite support, $\operatorname{Supp}(x)$ (which is always the case if we assume the axiom of excluded middle). Then $[\mathbb{A}]_{f} X$ is a path object.

Proof We write $r^{\prime}$ for the reflexivity map $X \rightarrow[\mathbb{A}]_{f} X$.

We will first define a map $g:[\mathbb{A}]_{f} X \rightarrow P_{Y} X$ such that the following diagram commutes.



Define

$$
X_{0}:=\{(x, a) \mid a \# f(x)\}
$$

We first define a morphism $g_{0}: X_{0} \rightarrow P_{Y}^{0} X$. We define $g_{0}(a, x)$ by induction on $|\operatorname{Supp}(x)|$. If $a \notin \operatorname{Supp}(x)$, then define $g_{0}(a, x):=x$. Otherwise, let $A:=\operatorname{Supp}(x)$, and note that $a \in A$. We define a 1 -open $A, a$-box $v$ as follows. Let $v(a, 0):=x(a:=0)$. For $\left(a^{\prime}, i\right) \in(A \backslash a) \times 2$, we may assume by induction that $g_{0}\left(\langle a\rangle x\left(a^{\prime}:=i\right)\right)$ has already been defined. Let $v\left(a^{\prime}, i\right):=g\left(a, x\left(a^{\prime}:=i\right)\right)$. We then define $g_{0}(a, x)$ to be $(v,(x(a:=0), x))$.

We now define $g(\langle a\rangle x)$ to be $g_{0}(a, x)(a:=1)$.

We also have a morphism $P_{Y} X \rightarrow[\mathbb{A}]_{f} X$ given by projection. This induces a morphism $K r \rightarrow K r^{\prime}$, which combined with the result before and the coalgebra structure on $r$ 
gives a commutative diagram.

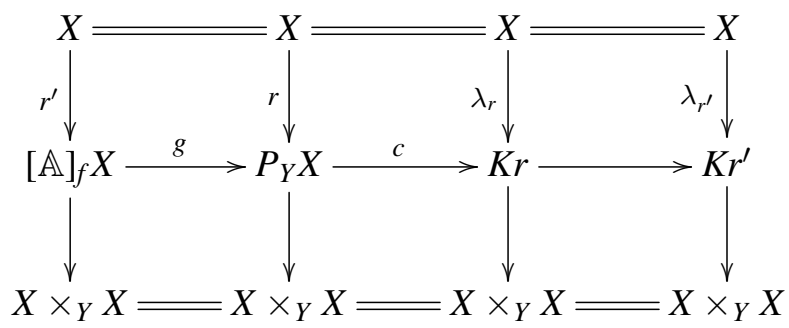

However, this easily gives us a coalgebra structure on $r^{\prime}$.

\section{Acknowledgements}

I am grateful for discussions with Nicola Gambino, Simon Huber and Christian Sattler, which were very helpful with this work. This work was carried out at the University of Leeds under EPRSC grant EP/K023128/1. I would like to thank the anonymous referees for their useful suggestions.

\section{References}

[1] S Awodey, MA Warren, Homotopy theoretic models of identity types, Mathematical Proceedings of the Cambridge Philosophical Society 146 (2009) 45-55; doi: 10.1017/S0305004108001783

[2] B van den Berg, R Garner, Topological and Simplicial Models of Identity Types, ACM Trans. Comput. Logic 13 (2012) 3:1-3:44; doi: 10.1145/2071368.2071371

[3] M Bezem, T Coquand, S Huber, A Model of Type Theory in Cubical Sets, from: "19th International Conference on Types for Proofs and Programs (TYPES 2013)", (R Matthes, A Schubert, editors), Leibniz International Proceedings in Informatics (LIPIcs) 26, Schloss Dagstuhl-Leibniz-Zentrum fuer Informatik, Dagstuhl, Germany (2014) 107-128; doi: 10.4230/LIPIcs.TYPES.2013.107

[4] E Bishop, D Bridges, Constructive Analysis, volume 279 of Grundlehren der mathematischen Wissenschaften, Heidelberg: Springer Verlag (1985)

[5] P Choudhury, Constructive Representation of Nominal Sets in Agda, Master's thesis, Robinson College, University of Cambridge (2015). Available at https://www.cl. cam.ac.uk/ amp12/agda/choudhury/choudhury-dissertation.pdf

[6] C Cohen, T Coquand, S Huber, A Mörtberg, Cubical Type Theory: a constructive interpretation of the univalence axiom (2015) arXiv:1611.02108 
[7] R Garner, Understanding the Small Object Argument, Applied Categorical Structures 17 (2009) 247-285

[8] M Grandis, W Tholen, Natural weak factorization systems, Archivum Mathematicum 42 (2006) 397-408

[9] S Huber, A Model of Type Theory in Cubical Sets, Licentiate thesis, University of Gothenburg (2015)

[10] E Palmgren, From intuitionistic to formal topology: some remarks on the foundations of homotopy theory, from: "Logicism, Intuitionism and Formalism - what has become of them?”, (S Lindström, E Palmgren, K Segerberg, V Stoltenberg-Hansen, editors), Synthese Library 341, Springer Netherlands (2009) 237-253

[11] A M Pitts, Nominal Sets: Names and Symmetry in Computer Science, volume 57 of Cambridge Tracts in Theoretical Computer Science, Cambridge University Press (2013)

[12] A M Pitts, An Equivalent Presentation of the Bezem-Coquand-Huber Category of Cubical Sets (2014) arXiv:1401.7807

[13] A M Pitts, Nominal Presentation of Cubical Sets Models of Type Theory, from: "20th International Conference on Types for Proofs and Programs (TYPES 2014)", (H Herbelin, P Letouzey, M Sozeau, editors), Leibniz International Proceedings in Informatics (LIPIcs), Schloss Dagstuhl-Leibniz-Zentrum fuer Informatik, Dagstuhl, Germany (2015)

[14] M Rathjen, Realizability for Constructive Zermelo-Fraenkel Set Theory, from: "Logic Colloquium '03”, (V Stoltenberg-Hansen, J Väänänen, editors), Association for Symbolic Logic (2006)

[15] E Riehl, Algebraic model structures, New York Journal of Mathematics 17 (2011) 173-231

[16] W P Stekelenburg, Realizability of Univalence: Modest Kan complexes (2014) arXiv: 1406.6579

[17] A W Swan, Some Brouwerian Counterexamples Regarding Nominal Sets in Constructive Set Theory (2017) arXiv:1702.01556

Institute for Logic, Language and Computation, Universiteit van Amsterdam, Amsterdam, Netherlands

a.w.swan@uva.nl

Received: 18 May $2016 \quad$ Revised: 31 December 2016 